\title{
A Study on Micro-scale Flow Behavior of Thermoplastic Polymeric Material
}

\author{
Takushi Saito, Tatsuya Kawaguchi and Isao Satoh \\ Department of Mechanical and Control Engineering, Tokyo Institute of Technology \\ 2-12-1, O-okayama, Meguro-ku, Tokyo 152-8552, Japan \\ tsaito@mep.titech.ac.jp
}

\begin{abstract}
In this study, two sets of experimental device were used to precisely investigate the thermal imprint process. The behavior of polymeric material transcribing the micro-structure of the stamp was observed by optical microscope, and effect of the temperature and pressure on the material behavior was discussed based on the obtained images. The obtained results showed that the temperature had larger effect on the transcription behavior. A generalized model that estimated the condition of the micro-scale transcription including the temperature dependence of the physical property of the material was also proposed. By using the model, the transcription ratio curves obtained at different temperature were successfully summarized on one master curve.
\end{abstract}

Keywords: Thermal imprint, Observational study, Temperature, Master curve

\section{Introduction}

Thermal imprint process is considered as simple and cost effective method. In the process, thermoplastic polymeric material is softened by heat so as to transcribe the mother pattern having micro-structure on its surface. The experimental study performed by Chou et al. is famous as a pioneer work of this method, and they transcribe nano-structure of the stamp to the polymeric material film [1]. This method has attracted researchers' attention since then, because it would become a promising mass-production process for precise and high value added products; such as optical and electronic elements, biotechnology and medical treatment usage.

In the thermal imprint process of polymeric material, the material behavior under processing is regarded as viscous or viscoelastic fluid. It is also expected that the interfacial effect is relatively increased in the scale of micron being the typical size of a thermal imprint product. There are several literatures that performed the experimental measurement and the numerical simulation about the final shape of the imprinted structure [2-8]. However, direct observation of the material behavior under the processing has not been studied enough, though it would provide a lot of useful information for the process improvement. And more, it is useful for the process optimization if the transcription behavior of the processing material against the micro-structure could be estimated quantitatively.

In this study, two sets of experimental device were constructed and the thermal imprint process was precisely investigated. The behavior of polymeric material transcribing the micro-structure of the stamp was observed by optical microscope, and the effect of temperature and pressure on the material behavior under the processing was investigated. A model including the temperature dependence of the physical property of the material was also proposed to estimate the progress of the micro-scale transcription.

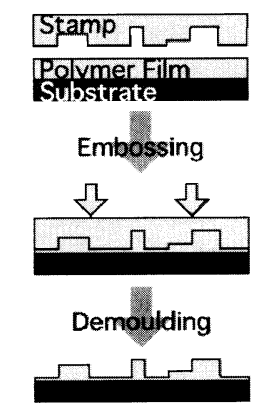

Fig. 1 Schematic process steps of the thermal imprint process

$\begin{array}{llr}\text { Received } & \text { April } & 5,2011 \\ \text { Accepted } & \text { May } & 13,2011\end{array}$




\section{Experimental Apparatus and Procedure}

Two sets of experimental device for thermal imprint process were prepared for the observational study. The first experimental device was used to precisely observe the polymeric material behavior during the process (Fig. 2). On the other hand, modeling of the transcription behavior to the micro-structure was examined by using the second experimental device (Fig. 3).

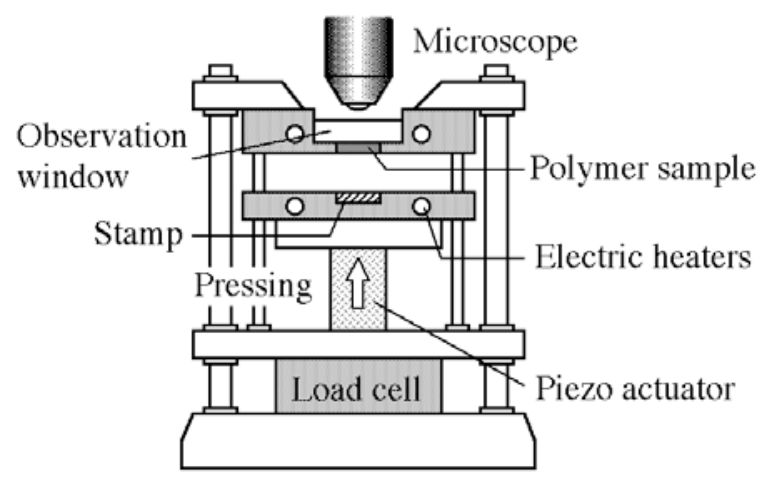

(a)

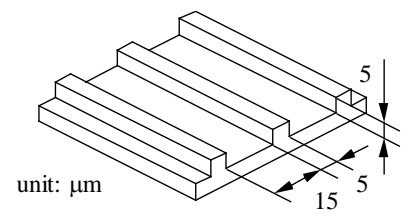

(b)

Fig. 2 (a) Experimental device for the precise observation of the polymeric material under the processing, (b) Surface geometry of the stamp

In the test section of the first device, the stamp having micro-structure is installed on the upper side of the lower plate, and the transparent window made of heat-resistant glass and the sample fixture are installed to the upper plate. Temperature of the upper and lower plates is controlled by the electric heaters. Micro-structure on the stamp surface was transcribed to the polymeric material by pushing up the lower plate driven by the piezo actuator. Pushing force under the transcription was measured by the force sensor installed between the actuator and the base-frame of the device.

Basic structure of the second device was almost the same as that of the first device. Stroke direction of the stage and its driving mechanism were modified from the first device so as to make the sample change easier. A pair of air cylinders was used to push the stamp against the polymeric material in good balance. The relationship between the provided air pressure and the pushing force of the transcription was calibrated by using a load cell. A n optical microscope that equipped a long focusing lens was used to observe the material behavior in the process in both cases. Process conditions used in the experiment are summarized in Table 1.

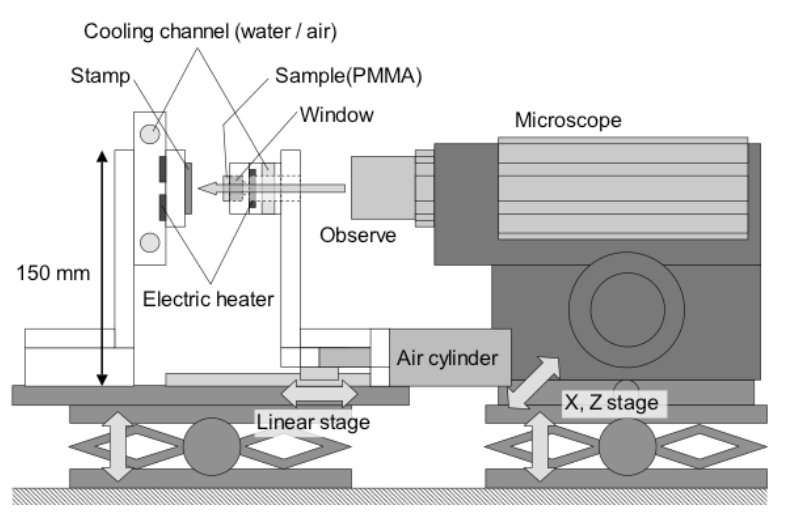

(a)

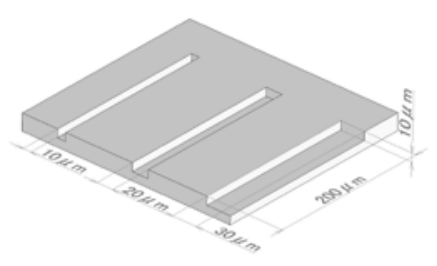

(b)

Fig. 3 (a) Experimental device for the modeling of the transcription behavior of the polymeric material, (b) Surface geometry of the stamp

Table 1 Process conditions used in this study

\begin{tabular}{|c|c|}
\hline $1^{\text {st }}$ experiment & $2^{\text {nd }}$ experiment \\
\hline \multicolumn{2}{|c|}{ Set temperature $\left({ }^{\circ} \mathrm{C}\right)$} \\
\hline $130-230$ & $130-180$ \\
\hline \multicolumn{2}{|c|}{ Pressure (M Pa) } \\
\hline $0.5-10$ & 1.2 (fixed) \\
\hline Compression time (min) \\
\hline 10 (fixed) & 1 (fixed) \\
\hline
\end{tabular}

$M$ icro-structure of the stamp was fabricated on a silicon wafer by using the inductively coupled plasma reactive ion etching (ICP-RIE) and its area size was $1.8 \times 1.8 \mathrm{~mm}$. In the experiment using the first device, straight groove structure with 15 $\mu \mathrm{m}$ width and $5 \mu \mathrm{m}$ depth was used (Fig. 2 (b)). A set of straight grooves with $10,20,30 \mu \mathrm{m}$ width, and $10 \mu \mathrm{m}$ depths was used in the second device (Fig. 3 (b)). In this study, no de-mold or lubricant agent was used for the stamp surface.

Tested specimen was made of poly(methyl methacrylate) (PMM A), and it was $20 \mathrm{~mm}$ length, 
$5 \mathrm{~mm}$ width, and $1 \mathrm{~mm}$ thickness. The specimen was dried for 24 hours before the experiment, and the transcription area was the center part of the specimen. Glass transition temperature of the PMMA was measured by differential scanning calorimetry, and it was approximately $103^{\circ} \mathrm{C}$.

\section{Results and Discussion}

3. 1. Observation of Transcription B ehavior (Effect of Temperature)

Observed images of the transcription behavior obtained by the first experiment is shown in Fig. 4. Two photos in the Figure correspond to the images observed at relatively low temperature $\left(130^{\circ} \mathrm{C}\right)$ and high temperature $\left(200^{\circ} \mathrm{C}\right)$, respectively. In the case of relatively low temperature, transcription of the polymeric material progressed in the longitudinal direction along the micro-structure at first, then it expanded slowly in the groove width direction with the passage of time. In the case of relatively high temperature, on the other hand, the transcription of the material gradually expanded from the part in where the surface of the material comes in contact with the surface of the micro-structure. As shown in these images, temperature dependence of the melt viscosity significantly influenced the transcription behavior of the material.

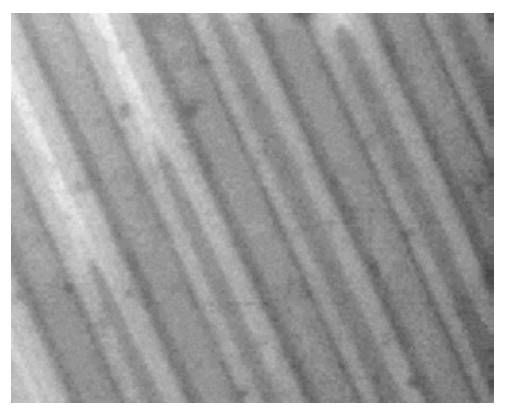

(a) Process temperature of $130^{\circ} \mathrm{C}$

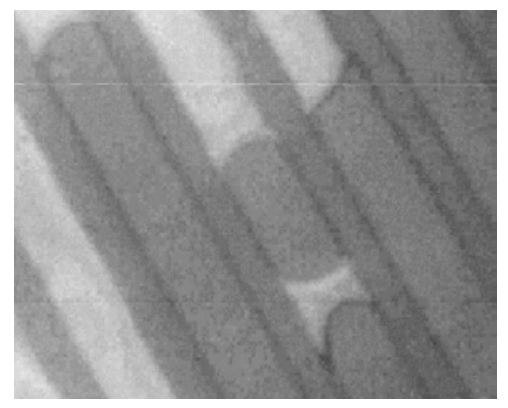

(b) Process temperature of $200^{\circ} \mathrm{C}$

Fig. 4 Observed polymeric material behavior transcribing the micro-structure (Pressure 0.5 $\mathrm{M} \mathrm{Pa}$ )
By using the obtained images through the observational study, effect of the temperature and pressure on the transcription behavior was investigated. As an index of the transcription level, width of the transcribed area W was defined (Fig. 5). Figure 6 shows the temperature dependence of $W$. Designed width of the micro-groove was $15 \mu \mathrm{m}$, and the measured width reached the designed value at around $200^{\circ} \mathrm{C}$. The meaning of this temperature was discussed in detail in the previous study [9].

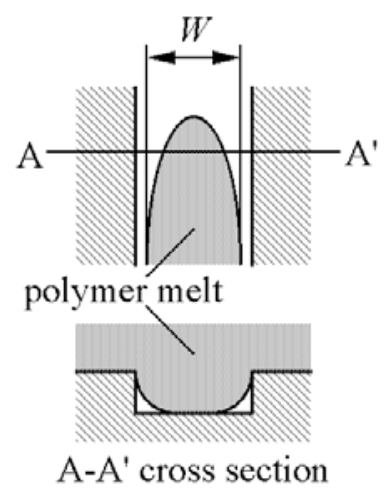

Fig. 5 D efinition of the melt front width of the observed image

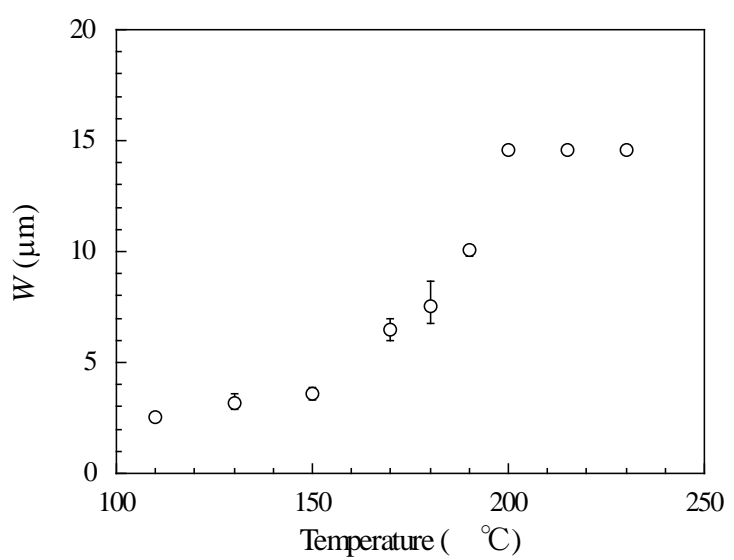

Fig. 6 Temperature dependence of the melt front width determined through the observed images

Time dependent change of the transcription progress was evaluated by using the image processing technique. Because the contacted interface between the polymeric material and the stamp surface showed dense color, the area of the dense color part was calculated as the representative value of the transcription progress. Figure 7 shows the result at the pressure of 0.5 $\mathrm{MPa}$. Required time for the transcription was significantly shortened by increasing the temperature. 


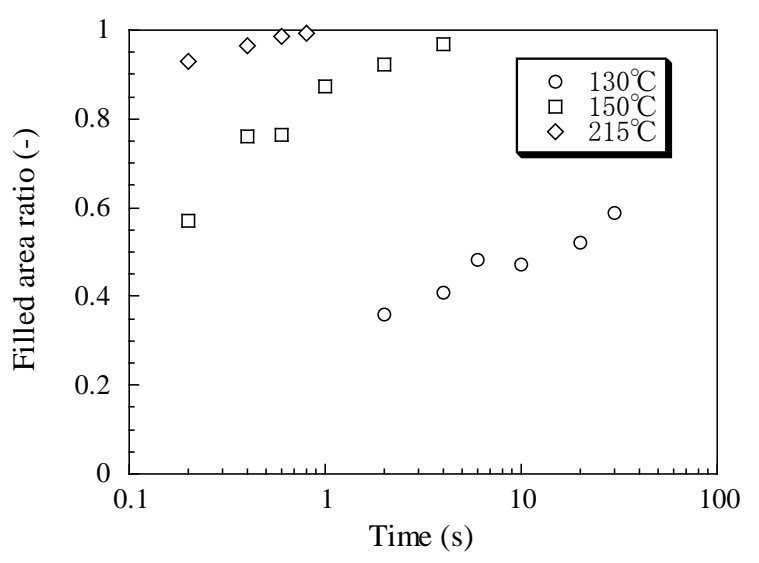

Fig. 7 Time dependence of the transcription progress at different temperature

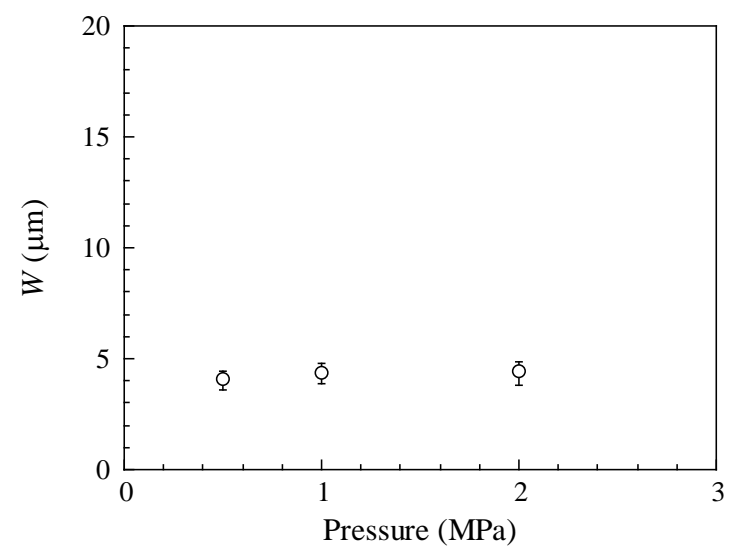

Fig. 8 Pressure dependence of the melt front width determined through the observed images

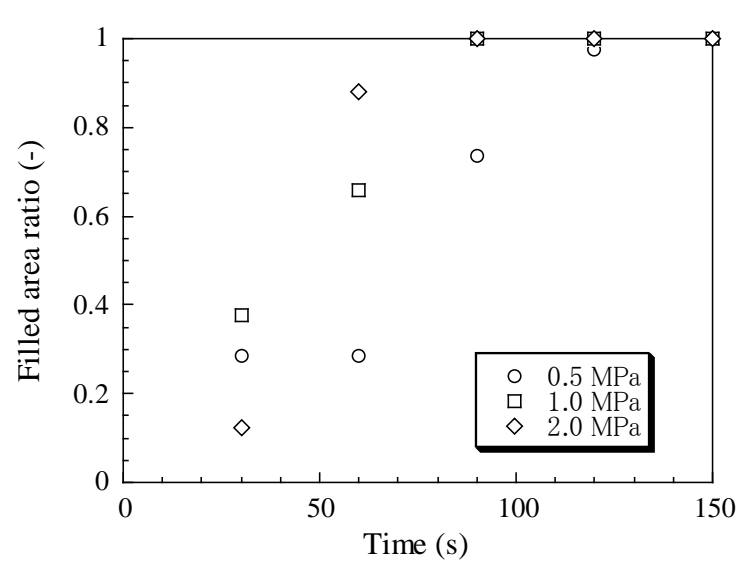

Fig. 9 Transcription progress with time at different pressure

\section{2. Observation of Transcription B ehavior (E ffect of Pressure)}

Figure 8 shows the pressure effect on the transcription width $W$ at the temperature of $130^{\circ} \mathrm{C}$. Despite the pressure variation, the transcription width hardly changed. Time dependent change of the transcription progress at different pressure is shown in Fig. 9. Effect of the pressure on the transcription behavior was obviously smaller than that of the temperature. This result indicated that the pressure did not have a large influence on the transcription time, especially in the case of relatively low temperature that is close to the usual thermal imprint process.

From the results obtained in above, a process strategy for the simultaneous realization of shorter processing time and better transcription was considered as follows; rising of the temperature at first, and then increasing the pressure moderately.

\section{3. Estimation M odel of Transcription}

The results obtained in the first experiment indicated that the transcription progress in the thermal imprint process was mainly varied by temperature. In this section, therefore, a simple model predicting the temperature effect on the transcription progress is introduced as a trial. Its validity is verified through the experiments performed by the second device.

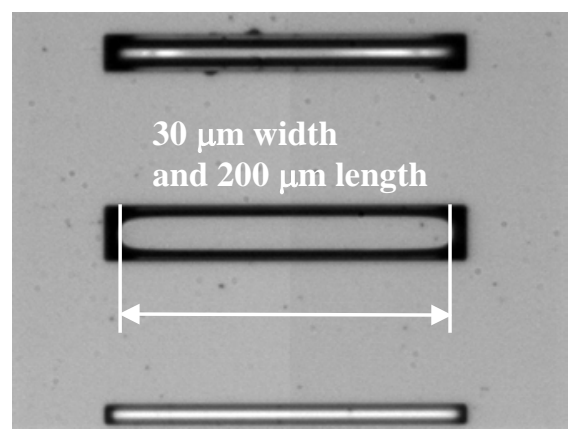

Fig. 10 Definition of the transcription length of the second experiment

The length of the contact area (lateral length in the rectangular groove) between the polymeric material and the stamp surface was quantified from the obtained images as shown in Fig. 10. The transcription progress in this section was discussed by measuring the transcription length (white arrow in Fig. 10). The filling ratio used in the following discussion is determined by dividing the measured length by the groove length of $200 \mu \mathrm{m}$.

Time dependent filling ratio at different temperature under the effective pressure of 1.2 $\mathrm{MPa}$ is shown in Fig. 11. The time required for the transcription was significantly shortened by increasing the temperature from $130^{\circ} \mathrm{C}$ to $180^{\circ} \mathrm{C}$. 


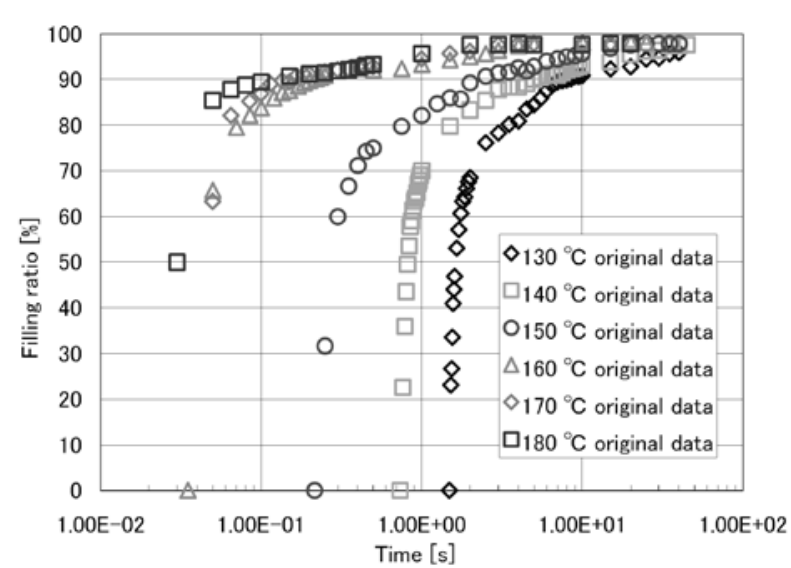

Fig. 11 Time dependent transcription progress at various temperatures

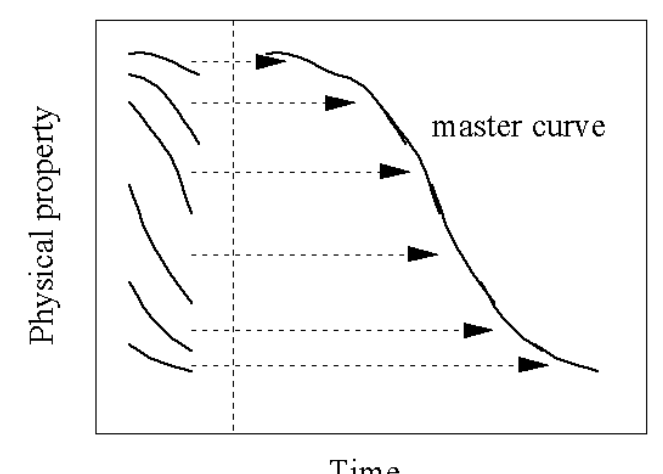

Fig. 12 Physical property (ex. stress relaxation modulus) measured at different temperature can be reduced to one master curve by appropriately shifting on the time axis.

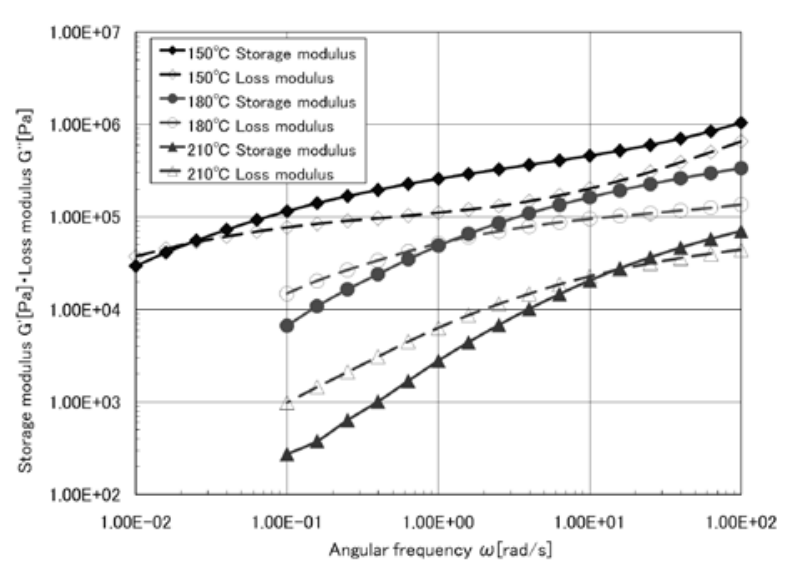

Fig. $13 \mathrm{M}$ easured storage modulus and loss modulus of the tested material

A function of transcription ratio $F(T, t)$ is defined here to generalize the effect of the temperature on the transcription behavior, and it represents the time dependent change of the ratio at the constant pressure $P$. There is a concept that is known as the time-temperature superposition by WLF equation [10]. The time-temperature equivalence is empirical relationship, and the stress relaxation data at different temperature would be reduced to one master curve for a reference temperature as schematically shown in Fig. 12. By using the similar hypothesis, the transcription ratio curves at different temperature were examined whether they form one master curve for a reference temperature.

Based on the concept described above, the transcription ratio change at temperature $T_{2}$ was converted into the ratio at temperature $T_{1}$ by using the shift factor $\alpha_{12}$ as follows.

$$
\begin{array}{r}
F_{1}\left(T_{1}, t_{1}\right)=F_{2}\left(T_{2}, t_{2}\right) \\
\text { where } t_{2}=\alpha_{12} t_{1}
\end{array}
$$

This equation means that the time axis is shifted by using $\alpha_{12}$ so as to superimpose the result of $\mathrm{F}_{2}$ on $\mathrm{F}_{1}$. Shift factor $\alpha_{12}$ that is important value in the model was calculated by the result of viscoelastic measurement performed to the polymeric material used in this study. Figure 13 shows the angular frequency dependence of the storage modulus and the loss modulus that was measured by the dynamic oscillation test. The value of cross-point of the storage modulus and the loss modulus $\omega_{c}$ was assumed as an inverse value of the relaxation time in this study. From the experiment, it was also suggested that a certain part of the pressure did not work for the micro-scale transcription but caused the bulk deformation of the polymeric material. Therefore, its effect was subtracted from the value of the pressure as $\mathrm{R}$ that was empirically determined in the experiment. Thus, $\alpha_{12}$ is given as follows.

$$
\alpha_{12}=\frac{\mathrm{P}-\mathrm{R}_{\mathrm{T}_{1}}}{\mathrm{P}-\mathrm{R}_{\mathrm{T}_{2}}} \times \frac{\omega_{\mathrm{CT}_{1}}}{\omega_{\mathrm{CT}_{2}}}
$$

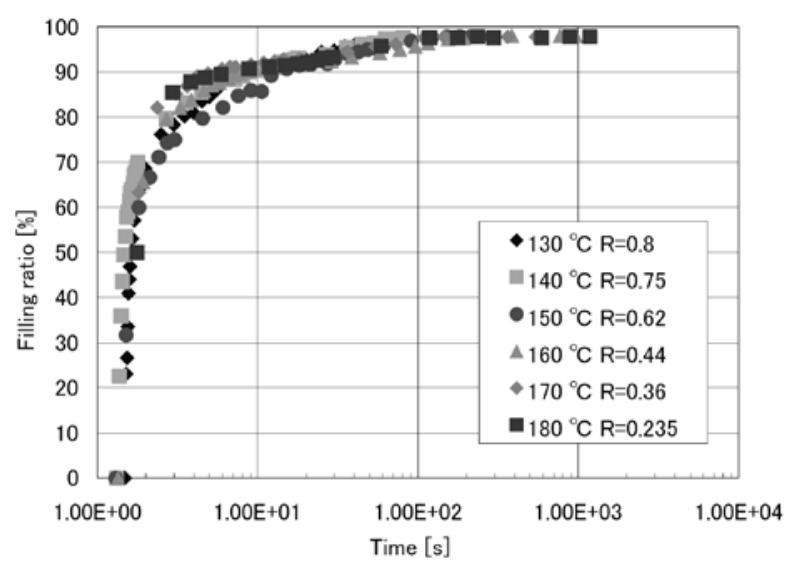

Fig. 14 The master curve of the transcription progress at the reference temperature of $130^{\circ} \mathrm{C}$ 
The data sets in Fig. 11 were shifted by using the equations discussed above so as to form the master curve at the reference temperature of $130^{\circ} \mathrm{C}$. Each curve of the transcription ratio change was successfully summarized on one master curve as shown in Fig. 14. From this result, it was concluded that the appropriateness of model describing the micro-scale transcription was verified.

\section{Conclusions}

In this study, the transcription behavior of the polymeric material in the thermal imprint process was directly observed by using optical microscope. The result showed that the temperature increase had large influence to shorten the transcription time, and the effect was superior to that of the pressure within the conditions investigated. It was also shown that the transcription progresses at different temperature settings were successfully superimposed on one master curve by using the concept of time-temperature superposition. This result was considered being useful to generalize the effect of temperature on the transcription behavior in the thermal imprint process.

\section{Acknowledgements}

The authors thank Mr. Atsushi Takata and Mr. Kenji Kodama for their help with experiments, and also thank Dr. Hiroshi Ito and Dr. Kazutoshi Yakemoto for their suggestions and discussions.

\section{References}

1. S. Y. Chou, P. R. Krauss and P. J. Renstrom, Applied Physics Letters, 67 (1995) 3114

2. L. J. Heyderman, H. Schifta, C. Davida, J. Gobrechta and T. Schweizerb, Microelectronic Engineering, 54 (2000) 229

3. T. Eriksson and H. K. Rasmussen, Journal of Non-Newtonian Fluid Mechanics, 127 (2005) 191

4. Y.-J. Juang, L. J. Lee and K. W. Koelling, Polymer Engineering \& Science, 42 (2002) 539

5. Y.-J. Juang, L. J. Lee and K. W. Koelling, Polymer Engineering \& Science, 42 (2002) 551

6. D. Yao, V. L. Virupaksha and B. Kim, Polymer Engineering \& Science, 45 (2005) 652

7. H. Takagi, M. Takahashi, R. Maeda, Y. Onishi, Y. Iriye, T. Iwasaki and Y. Hirai, Microelectronic Engineering, 85 (2008) 902

8. Y. Onishi, Y. Hirai, H. Takagi, M. Takahashi, T. Tanabe, R. Maeda and Y. Iriye, Japanese Journal of Applied Physics, 47 (2008) 5145

9. T. Saito, T. Kawaguchi and I. Satoh, IEEJ Transactions on Sensors and Micromachines, 130 (2010) 356

10. M.L. Williams, R.F. Landel and J.D. Ferry, Journal of the American Chemical Society, 77 (1955) 3701 